\title{
Sustainability and Interoperability: Two Facets of the Same Gold Medal
}

\author{
Michele Dassisti ${ }^{1}$, Ricardo Jardim-Goncalves ${ }^{2}$, Arturo Molina ${ }^{3}$, Ovidiu Noran ${ }^{4}$, Hervé \\ Panetto $^{5,6}$, Milan M. Zdravković ${ }^{7}$ \\ ${ }^{1}$ DIMEG, Politecnico di Bari, Italy, ${ }^{2}$ FCT, UNINOVA, Portugal, \\ ${ }^{3}$ Tecnológico de Monterrey, Mexico, ${ }^{4}$ School of ICT, Griffith University, Australia, \\ ${ }^{5}$ University of Lorraine, CRAN UMR 7039, France, ${ }^{6}$ CNRS, CRAN UMR 7039, France, \\ ${ }^{7}$ LIPS, Faculty of Mechanical Engineering, University of Niš, Serbia \\ 1'm.dassisti@poliba.it, ${ }^{2}$ rg@uninova.pt, ${ }^{3}$ armolina@itesm.mx, ${ }^{4}$ o.noran@griffith.edu.au, \\ ${ }^{5,6}$ herve.panetto@univ-lorraine.fr, ${ }^{7}$ milan.zdravkovic@masfak.ni.ac.rs
}

\begin{abstract}
To sustain is to endure' - that is, to be able to survive and continue to function in the face of significant changes. The commonly accepted concept of 'sustainability' currently encompasses three main pillars: environmental, social/ethical and economic. In a metaphor of survival, they can be seen as water, food and air; one needs all three, only with varying degrees of urgency. In today's globally networked environment, it is becoming obvious that one cannot achieve environmental, social or economic sustainability of any artefact (be it physical or virtual, e.g. enterprise, project, information system, policy, etc) without achieving ubiquitous ability of the artefact and its creators and users to exchange and understand shared information and if necessary perform processes on behalf of each other - capabilities that are usually defined as 'interoperability'. Thus, sustainability relies on interoperability, while, conversely, interoperability as an ongoing concern relies for its existence on all three main pillars of sustainability. This paper aims to test the hypothesis that interoperability and sustainability are two inseparable and inherently linked aspects of any universe of discourse. To achieve this, it applies the dualistic sustainability / interoperability viewpoint to a variety of areas (manufacturing, healthcare, information and communication technology and standardisation), analyses the results and synthesizes conclusions and guidelines for future work.
\end{abstract}

Keywords: Sustainability, Interoperability, Manufacturing, Information and Communication Technology, Health Informatics, Standardisation

\section{Introduction}

History has shown that the continued existence of businesses depends not only on their economic sustainability but also on their impact on the natural environment and the way they treat their workers. This basic truth was emphasized by Elkington's [1] Triple Bottom Line (TBL) approach to business sustainability: one must achieve not only economic bottom-line performance but also environmental and social accomplishment. In Blackburn's [2] vision, we can compare economic sustainability 
to air and environmental and social sustainability to water and food: the first is more urgent but not more important than the others - hence, any successful enterprise must take a whole-system approach to sustainable development [3]. In today's globally networked environment, one cannot achieve environmental, social / ethical or economic sustainability of any artefact (be it physical or virtual, e.g. enterprise, project, information system (IS), policy, etc) without achieving ubiquitous ability of the artefact and its creators and users to exchange and understand shared information and if necessary perform processes on behalf of each other - in other words, interoperate. Thus, sustainability relies on interoperability, while, conversely, interoperability as an ongoing concern relies on all three main pillars of sustainability.

This paper aims to test the hypothesis that interoperability and sustainability are two inseparable, balanced and inherently linked aspects of any universe of discourse (see Fig. 1) by applying the proposed dualistic viewpoint to a variety of areas, analysing the results and synthesizing conclusions and guidelines for future work.

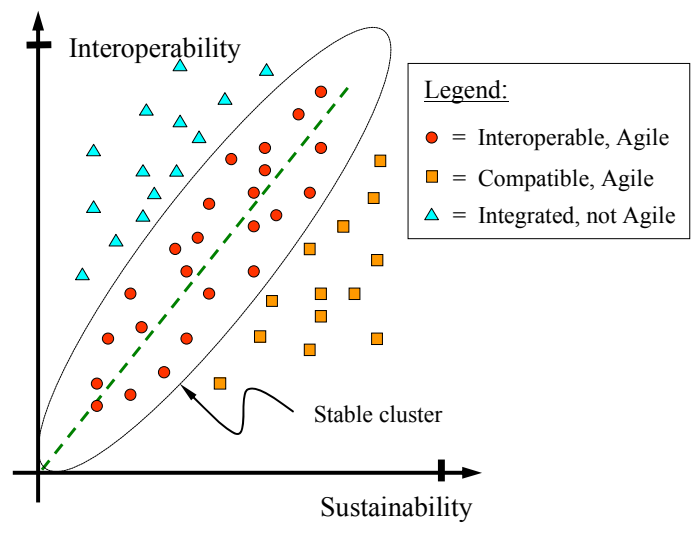

Fig. 1. The Sustainability - Interoperability continuum

\section{Sustainability and Interoperability}

Interoperability is typically defined as the ability of systems or components to exchange and use information [4]. ISO 16100 [5] defines manufacturing software interoperability as "the ability to share and exchange information using common syntax and semantics to meet an application-specific functional relationship [..]". Thus, generally speaking, interoperability is the ability to work together effectively and to exchange information in a useful and meaningful way [6].

While the term 'sustainability' is nowadays encompassing ecological, economical and ethical concerns, the focus in on the results, rather than on the means to achieve them. Thus, the main question is: Can one improve the sustainability of a given system by enhancing its capability to interoperate with its environment? The difference between integration and interoperability has been explained in ISO 14258 [7], who describes three ways in which models could be related: (1) integration, with 
a standard or pivotal format to represent these models; (2) unification, with a common meta-level structure for semantic equivalence between these models; and (3) federation, when each model exists per se, with the mapping between concepts done at an ontology level to formalise interoperability semantics.

Integration is generally considered to involve a degree of functional dependence and hence less flexibility. Integration also deals with organisational issues, albeit in a less formal manner owing to the human factor. Compatibility is something less than interoperability, where systems/units do not interfere with each other's functioning but are not able to exchange services. To conclude, interoperability lies in the middle of an 'Integration Continuum' between compatibility and full integration [6]. It is important to distinguish between compatibility, interoperability and integration in order to have a meaningful debate on how to achieve them.

The question is: is one able to make a similar analysis and demonstration relating to the three main facets of sustainability, i.e. Economic, Ecologic, Ethical / Social?

From an Economical perspective, interoperability is an a priori property for the socalled 'Network effect' [8]. For larger networks, interoperability is a competitive weapon; they have nothing to gain by interoperating and thus their best strategy is to drive organic growth while waiting for smaller networks to 'wither on the vine'. However, the mid-sized networks can gain market leadership by interoperating with each other; so the risk of this happening has to be weighed by the market leaders. In order for a market leader to establish an insurmountable lead, they would have to create interoperability with a smaller network (ideally the number 2 network) such that the combined value of all remaining competing networks would not be a threat. In this way, interoperability is contributing to the sustainability of the systems that need a capacity to interoperate by improving their economic competitiveness.

From an Ecological perspective, interoperability is contributing to the so-called 'Green Information Technology (IT) ', which is a complex and still 'fuzzy' concept, partly due to the complexity and diversity of the IT applications and development. The goal of the IT-related economic development is differs regionally; for example, the European Union countries have devised a plan of 'Green Knowledge Society' [9] to help promoting the application of the Green IT, since knowledge is the dominant element in the economic structure of EU. Green IT is especially useful for the improvement of the green production mode and the supervisory control in traditional industries, the reduction of the pollution emissions and the energy utilization. Recent research results suggest that when ICT is applied in other industries the amount of the energy saving is 5 times that of its own. Reducing the heterogeneity, improving the quality of data stored by IS and assessing the risks of poor interoperability [10] can help ecological sustainability of those interoperable systems.

From an Ethical perspective, interoperability is concerned with data privacy, information protection, trust and access control. Policy makers recognize the need for robust, protected data flows if the benefits of an information economy are to be realized. While the global flow of data is essential for innovation and economic growth, companies face significant challenges when attempting to comply with often conflicting requirements of diverse national and regional data protection laws and regulations. Emerging policies stress the need to create a streamlined system which allows for the smooth movement of data across regimes, in a manner that ensures that individuals and enterprises enjoy the protection afforded by local laws and regulations 
[10]. That is the conundrum of sharing information: the information is essential to improve collaboration towards an economical benefit; however, on the other hand, sharing it presents the risk of possible loss, knowledge theft and protection rights.

\section{A Sustainable Interoperability of Standards}

Continuous change processes enable enterprises to endure in today's competitive and highly dynamic business ecosystem; however, they also bring about new challenges, such as the potential weakening of internal and external interoperability displayed by the enterprise. Data, application and business process [11] at technical, syntactic, semantic and pragmatic levels [12] may all be affected by change. This problem can occur within enterprises but also between members of collaborative networks (CNs) [13]. The conceptual syntactic aspects of interoperability are typically addressed by agreeing on and upholding standardised formats to overcome barriers [14, 15]. Unfortunately however, the standards describing such formats are themselves plagued by interoperability problems and are in fact just another facet of the interoperability challenge inherently posed by mandated continuous change processes.

Currently, it appears that many Technical Committees (TCs) and Sub-Committees (SCs) do not have a holistic, birds-eye view of the standards they administer and develop, mainly due to the lack of a proper central standards repository. In addition, the visibility between the custodian Work Groups (WGs) within TCs and SCs is poor, which leads to potential gaps, overlaps and inconsistencies in standards development. As the mandatory review process occurs in an asynchronous way for each standard, all stakeholder WGs (not just the custodian) should be aware of and have input in the proposed changes. However, in reality the involvement of other potential stakeholders in the development or maintenance of a standard occurs on an irregular and anecdotal basis, relying on the goodwill and knowledge owned by the WG conveners, librarians etc. Hence, changes to a standard (glossary, procedures, etc) do not automatically notify or propagate to other relevant WGs and standards they administer.

Typically, the setup and operation of any project requires a set of standards. Currently, it is quite difficult for the typical user to readily find out what standards are required for a particular type of project as the user guides do not explain how to use standards together. Furthermore, the terminology inconsistency, gaps and overlaps of the standards selected for a project confuse the users and end up affecting the sustainability and interoperability of the enterprise(s) attempting to use the standards. Thus, current interoperability of standards is itself low and unsustainable.

Based on the previous results and ongoing research work [16, 17], we propose a structured and widely applicable approach to achieve sustainable interoperability, consistence and redundancy elimination. Thus, a common workspace based on a Structured Repository (SR) would be a good start to address the problems identified.

Interoperability-relevant SR elements (aspects, barriers etc) can be sourced by mapping and decomposing the mainstream interoperability frameworks and relevant standards in respect to a reference framework (e.g. GERA, in [18]).

Rules can then be added to enable querying the SR and thus transforming it into a knowledge base (see Fig. 2 and [17] for details), as part of an expert system guiding 
standards creation and review in an integrated, collaborative and synergistic manner.

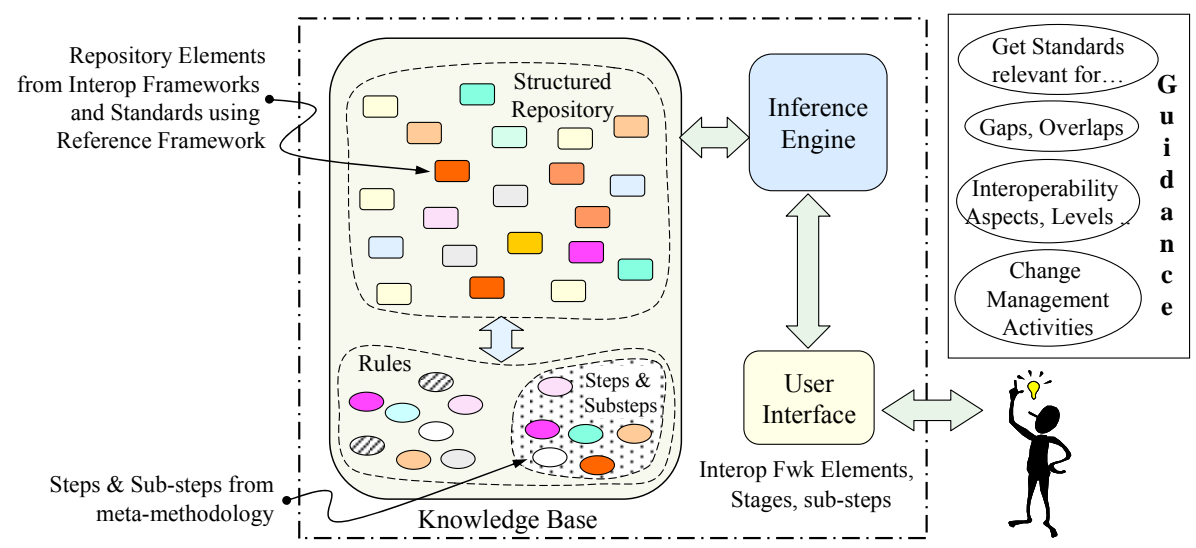

Fig. 2. Proposed expert system supporting a sustainable interoperability of standards

The user employs the inference engine to elicit knowledge such as: what standards cover a specific area; is there a need for a new standard, to retire or to change a standard (any overlaps?); what are the ripple effects of a standard's revision; what are the activities to create / review / retire standards, etc. The expert system responds with ranked solutions on views, aspects, levels, development activities, tools, etc.

Standards interoperability is essential in promoting user acceptance, usability and efficient administration and maintenance; however, it is also a complex undertaking involving a multitude of aspects and levels, from infrastructure to politics and organisational culture of the custodian work groups. The proposed system provides a foundation towards a sustainable interoperability of standards - a benefit that will propagate to the enterprises and collaborative networks using the standards.

\section{Sustaining Interoperability of Electronic Healthcare Records}

The current fragmentation of health science along traditional boundaries is considered as inefficient, particularly when considered in the contemporary patient management context. The Virtual Physiological Human (VPH) is intended to address this issue by providing a framework that enables the integration of data and observations about human's biology. Today, VPH can be specialized to a subset of data, which is manageable in current healthcare settings. This subset of VPH data is an Electronic Health Record (EHR), defined as a longitudinal electronic record of patient health information generated by one or more encounters in any care delivery setting.

Sustainability of EHR is directly related to sustaining their interoperability, namely towards exhibiting the robustness to a change of environment in which EHR is used. That is, EHR is sustainable as long as different clinical IS can operate in the same way (or draw the identical conclusions) based on a single EHR; or, EHR is 
sustainable as long as the same clinical IS can operate in the same way on basis of the different representations of same EHR data.

One of the related problems is that terminologies are not bound to EHR standards. Rector et al [19] used ontology to bind HL7 messages to SNOMED-CT (Systematized Nomenclature of Medicine - Clinical Terms) codes; however, the semantic interoperability between the systems using the above standards is still an issue. The diversity of medical ontologies based on various conceptualization approaches makes mapping difficult. Two development approaches are 1) bottom-up formalization of the vocabularies and 2) semantic analysis of the clinical care and development of facilitating ontologies. Although the first approach is dominant in the research community, Beale and Heard [20] argue that the starting point for a successful model is an ontological analysis of the healthcare delivery process.

In fact, the diversity of conceptualization choices is not necessarily an issue. Bottom-up approaches and narrow scopes are characterized by decreased development time and thus, they contribute to the commoditization of the semantic technologies in a medical sector. The difficulties of integrating such ontologies (caused by implicitness) can be resolved by using upper ontologies. Schulz et al [21] addressed logical and ontological issues of SNOMED; the well-known Ontology of Biomedical reality [22] was developed as a result of the process of vertical integration of upper ontology (Basic Formal Ontology - BFO) and domain ontology. Although this approach introduces new artefacts into the context, the sustainability of an integrated knowledge is actually being increased. Due to its general approach in describing the world, one of the key features of the upper ontologies is the resilience to change. When formal models are considered, this resilience decreases with the used level of abstraction in their development. Similar conclusion can be drawn for the increase of the implicitness level, which is notable for more specialized ontologies. When upper ontologies are used in correlating different standards, the total number of mappings decreases. Hence, the effort needed for their maintenance is reduced.

Partitioning can also help the maintainability of the ontological framework. Besides the computational performance benefits, this decoupling allows developers to compensate for these failings without compromising the underlying models.

Clearly, one approach to achieve sustainability would be to identify and maintain the mappings between the concepts of the different EHR standards in their native forms. However, this approach itself would not be sustainable because of decreased scalability of such mappings and high maintenance effort required. Another approach is related to defining and associating the formally defined meanings to the EHR concepts. Hence, the robustness of EHR would be drawn from the capability of systems to interpret the semantic similarities or differences of the EHR concepts, based on their formal representations. The inference capability of these systems would allow the logical correspondences between two representations to be computed on the basis of the relationships between the concepts of the respective representations and the concepts of formal ontologies. 


\section{Virtualisation: addressing Interoperability and Sustainability in Distributed Manufacturing}

Manufacturing can be defined as a process composed of activities performed using various resources and aimed at transforming a form of matter into another for a given scope (typically responding to a set of customer requirements). There is significant scientific interest in making manufacturing sustainable, as unfortunately it is currently one of the most important causes of global resource depletion. This challenge is compounded by the current distribution of activities and processes; this requires interoperability of the activities along the distributed manufacturing chain.

As a reaction to this challenge, the new paradigm of Virtual Manufacturing (VM) has emerged in the last decades as an improved capability of sharing and managing knowledge and synchronizing cooperation activities in a form useful to the human decision factors. The improvements in sensor technologies (hardware) as well as in simulation facilities (software) have been the key driver of this 'soft revolution', making the use of IT and computer simulation more effective in modelling real world manufacturing processes for the purpose of understanding and controlling them [23].

The origins of virtual reality can be traced as far back at least as 'the ultimate display' [24], allowing a user to 'step through' the computer screen into a threedimensional (3D) world. Thus, with the advance of computer technology, VM turned into a way to reduce the time and cost involved in decision making and simulation (and thus covering the entire product lifecycle, from conceptualization to production). The user can look at, move around, and interact with the virtual world as if they were real. An essential question is how and to what extent could VM (as a breakthrough technology to support interoperability in manufacturing) contribute towards achieving sustainability along the manufacturing chain?

Given the scarcity of bibliographical references, a possible viewpoint to address this new question is to refer to the very nature of VM, which is in fact synonymous to simulation of the manufacturing processes. Simulation is defined as experimentation (using some model) on a system of interest, whether existing or not [25]. Simulation helps reduce uncertainty in the decision making process by allowing to explore several alternatives with relatively low costs and by presenting knowledge about projected artefacts on various levels of abstraction. In other words, when dealing with VM one adopts a computer system to generate information about the structure, status, and behaviour of a manufacturing system (whether existing or not) as if it was in a real setting, i.e. to provide a capability to 'manufacture in the computer' [24]. In this context, the VM connection to sustainability becomes obvious; VM has the potential to provide a modelling and simulation environment allowing the sustainability of fabrication/assembly of any product, including the related manufacturing processes, to be assessed by computer simulation. For example, one can in measure the energy required to manufacture, or can estimate the amount of material and its footprint from well-known databases (e.g. [26, 27]), or even foresee potential improvements by adopting different materials. Furthermore, it can be shared with other suppliers along the distributed manufacturing chain, even in absence of a predetermined standard.

In this context, a new concept can be introduced, Virtual Sustainability (VS) that can be defined as feature of a product/process obtained by the grace of the above-said 
virtual paradigm. Provided adequate tools are available, one can assess the sustainability of processes or products before any concrete action is taken, in a preconceptual stage and independently of the manufacturing chain stage. It is only a matter of the quality and power of modelling and simulation tools, techniques and applications to offer new opportunities to evaluate the impact on resource consumption and environment because of these features. Virtual sustainability then reflects into 'real sustainability' as products or processes implemented in the real world can be improved to address economical, environmental or even social pillars, since VM application can encompass the entire life cycle of a product or process.

In order to clarify the VS concept, one can refer to three stages of representation of knowledge and information of an entity according to [28]: digital, virtual and real. A digital entity is the representation of the digital information, such as a knowledge model of the entity. The virtual representation of an entity is its representation within a domain for validation purposes, where the link between digital and virtual can be a reference architecture. Finally, the real entity is the implemented actual test scenario and thus reflects the former stages. Whatever the entity we are reasoning about (be a manufacturing system, a process, a product) or its features related to sustainability, it is quite clear that virtualisation is simply a indissoluble part of the same continuum formed by interoperability and sustainability before recalled in Fig. 1.

\section{Control Systems for Sustainability, Sustainable Control Systems}

In the context of the fundamental changes to environmental, social and lifecycle requirements we currently have to deal with, the legacy isolated control system concepts have to be overlapped in order to create better systems where the concept of boundaries disappear [29]. The following shows the control techniques basic laws that are used in Control Systems for Sustainability and Sustainable Control Systems.

Thus, Intelligent control uses various Artificial Intelligence computing approaches; Optimal control is a particular control technique in which the control signal optimizes a certain cost index; Robust control deals with uncertainty in its approach to controller design; Adaptive control uses on-line identification of the process parameters, or modification of controller gains; Hierarchical control arranges a set of devices and governing software in a hierarchical tree; Stochastic control deals with control design with uncertainty in the model and Energy-shaping control views the plant and the controller as energy-transformation devices.

The basic considerations for defining Control Systems for Sustainability and Sustainable Control Systems are presented in a new control system concept named Sustainable Control System for Sustainability (SCSFS). This design has to include the primary and secondary factors that are presented below [30, 31]:

1. Defining the control systems limits by the carrying capacity of the environment.

2. Taking account the effect o the social necessities

3. Respecting life forms and supports biodiversity in the design; accidents leading to environmental concerns are key focus areas for governments and public alike; 
4. Employing ecological decision-making systems inside the control laws;

5. Creating balanced, open and flexible control laws that incorporate the social, economic and environmental factors as a unique solution;

6. Using global control efforts and natural resources;

7. Appling renewable and reliable sources of energy in the control system;

8. Eliminating harm to the environment from the controller;

9. Designing control systems that use long lifetime cycles;

10. Guarantee the sustainability of the controller during a long period of time for future control systems developments;

11. Employing economical decision-making systems inside the control system;

12. Developing a self-categorization control system, including methodologies for lifetime cycle stages;

13. Utilizing the lifetime cycle stages as a dependent variable in the controller;

14. Generating a positive impact on the life cycle during early control design stage;

15. Developing an integrated control system that includes a cost management function. This is because non-common operating point and maintenance controller strategies are rarely planned at the design phase, so those could affect the lifetime.

16. Adopting new controller technologies to maintain competitive advantage.

The concept of life cycle is a key factor for designing Control Systems for Sustainability and Sustainable Control Systems. It is important to include all the aspects of life cycle: economical, environmental, social, and society, but also controller performance, scalability, reconfiguration and integration. The intention of controls systems for sustainability is to eliminate negative environmental impact completely through skilful, sensitive and sustainable control design and minimum impact on the environment during system operation. The control system concepts are included in the process design for improving the overall performance of the system.

It is expected that the proposed SCSFS based on the above-mentioned principles and concepts will provide suitable solutions for the challenges posed by the next generation of control systems.

\section{Sustainability and Interoperability of Negotiation Processes}

In a globalised and networked market, businesses face the struggle to perform better and faster, using new approaches and techniques and optimising their way in order to stay in business. In the light of this highly competitive and demanding environment, the establishment of strategic partnerships and outsourcing are common practices that frequently compete with in-house development. To reach a sustainable operation, companies need to continuously negotiate to create and maintain interoperability supported by an appropriate knowledge management framework [32, 33].

Negotiation of tasks and jobs implementation is therefore a task that plays a crucial part on its business evolution. Negotiations often involve several heterogeneous parties, external and internal, using different tools, languages, platforms and Information and Communication Technologies (ICT); hence, several internal company areas compete or collaborate with external parties for the development of a project, or outsource parts of it. Best practices specify that decision analysis must 
convey a thorough documentation, evaluation and analysis of the alternative solutions in order to reach the best solution and learn from it. On the other hand, the various parties involved in the negotiation need to be interoperable, sharing business, technical data and information seamlessly.

This requires a framework for sustainable interoperability of negotiation processes, using formal description (modelling) to support the coordination of multiple parallel negotiations occurring in business-to-business interactions and offering mechanisms to support negotiations in a distributed environment. This includes a set of hierarchically layered and distributed components that implement the rules of the modelled negotiation and also handle the interoperability aspects of the negotiation.

A sustainable, flexible and generic approach towards the implementation of the underlying infrastructure can be provided by a Cloud web-service-based platform (e.g. Software as a Service, SaaS). Such infrastructures and service provider platforms allow subscribing to processing that matches the negotiation environment needs.

The framework's top layer (Negotiation Manager) is targeted to the Manager of each negotiation party. It handles all business decisions [34] that need to be taken (e.g. proposal, acceptance of proposal, rejection of proposal, invite of another party to take part in the negotiation) and analyses and manages the negotiated parameters, communicating with the lower layers using web-services [35].

A second layer is dedicated to the Coordination Services (CS) which assist the negotiations at a global level (negotiations with different participants on different jobs) and at a specific level (negotiation on the same job with different participants) handling all issues regarding communication at this layer level. The CS shall also handle the on-going transactions and manage the persistence for the status of the negotiation sequences. To improve the interoperability, data shall be exchanged using the standard protocol ISO10303 STEP [35]. This layer is also responsible for handling semantic discrepancies between the negotiating parties via the use of one or more ontologies and may include an agent-based architecture to support the complexities of the negotiation operations through the Middleware layer.

These middleware services shall provide support for performing all aspects related with basic infrastructure, and handling the heterogeneity related with multiple negotiation players; it may also include publication of the job requirements and characteristics, in order to allow potential companies interested in participating to 'subscribe' to it and be able to enter the negotiation.

Each negotiation is organised in three main steps: initialisation; refinement of the job under negotiation; and closure. The initialisation step allows to define what has to be negotiated (Negotiation Object) and how (Negotiation Framework) [36]. In the refinement step, participants exchange proposals on the negotiation object trying to satisfy their constraints. Closure concludes the negotiation.

To manage all issues regarding semantics between the negotiating parties, an ontology can handle generic negotiation terms not bound to any specific business, as well as using dedicated ontologies to handle semantics related to negotiated items. To foster a higher independence of external factors, the services and infrastructure are modelled in a Model-Driven Architecture (MDA) Platform-Independent Model (PIM) [37] defining the basic foundations of the framework, which is then transformed into the final Platform-Specific Model (PSM) set of services [38]. 


\section{Conclusions}

This paper has attempted to demonstrate that sustainability and interoperability are intertwined aspects of any universe of discourse. After clarifying and structuring the relevant concepts and areas of these two aspects, we have described several areas of research and practice where the connection between sustainability and interoperation is manifest and we have proposed solutions to existing or emergent problems.

Thus, in the Standardisation area we have analysed the current issues and proposed a system towards improving standards and custodian Work Groups' interoperability in a sustainable manner. In the Health Informatics domain we have analysed existing problems and proposed some improvements to Electronic Health Records sustainability related to their formal representations. In Manufacturing we have explained the virtualisation concept, proposed the Virtual Sustainability model and argued its benefits on the interoperability and sustainability of global manufacturing. In Control Systems we have defined the laws and considerations for sustainable control systems and have explained the merging of control systems, interoperability and sustainability concepts in a Sustainable Control Systems for Sustainability paradigm. In Negotiations we have explored the need for interoperability in the global negotiations required to sustain a business and we have proposed a cloud-based, agent-driven multi-layered negotiation framework providing flexible semantic interoperability.

To sum up, in a global networked environment deeply affected by financial crises, climate change and pandemics, the necessary economic, environmental and social / ethical sustainability cannot be achieved without sustainable interoperability.

\section{References}

1. Elkington, J, Cannibals with Forks: The Triple Bottom Line of $21^{\text {st }}$ Century Business. 1998.

2. Blackburn, W.R., The Sustainability Handbook. 2007, Cornwall, UK: EarthScan Publish.

3. UN World Commission on Environment and Development, Our Common Future (Brundtland Report). 1987, Oxford: Oxford University Press.

4. IEEE, IEEE Std 610.: Standard Glossary of Software Eng. Terminology. 1990: p. 1-84.

5. ISO, ISO1 16100-1:2009 Ed.2: Industrial automation systems and integration Manufacturing software capability profiling for interoperability - P 1: Framework. 2009.

6. Panetto, H., Towards a classification framework for interoperability of enterprise applications. Int. J. of Comp. Integrated Manufacturing, 2007. 20: p. 727-740.

7. ISO, ISO14258 Industrial Automation Systems Concepts \& Rules Enterprise Models. 2005.

8. Robinson, C.K., Network Effects in Telecommunications Mergers-MCI WorldCom Merger: Protecting the Future of the Internet, in allocution devant le Practicing Law Institute. 1999

9. Forge, S., et al., A Green Knowledge Society-An ICT policy agenda to 2015 for Europe's future knowledge society, Ministry of Enterprise, Government Offices of Sweden, 2009.

10. Yahia, E., A. Aubry, and H. Panetto, Formal measures for semantic interoperability assessment in cooperative enterprise information systems. Comp Ind, 2012. 63: p. 443-457.

11. IDEAS. IDEAS Project Deliverables (WP1-WP7), Public reports. 2003 [cited 2011 Jul]; Available from: www.ideas-roadmap.net.

12. Whitman, L. and H. Panetto, The Missing Link: Culture and Language Barriers to Interoperability. Annual Reviews in Control, 2006. 30(2): p. 233-241. 
13. Noran, O., Collaborative networks in the tertiary education industry sector: a case study. International Journal of Computer Integrated Manufacturing, 2012. 26(1-2): p. 29-40.

14. Chen, D. Framework for Enterprise Interoperability 2006 [cited 2011]; http://www.finescluster.eu/fines/jm/Download/53-Framework-for-Enterprise-Interoperability-Chen.html.

15. Noran, O. and P. Bernus, Effective Disaster Management: An Interoperability Perspective. Lecture Notes in Computer Science, 2011. 7046: p. 112-121.

16. Noran, O., A Meta-methodology for Collaborative Networked Organisations: Creating Directly Applicable Methods for Enterprise Eng Projects. 2008, Saarbrücken: VDM Verlag

17. Noran, O., Achieving A Sustainable Interoperability of Standards. IFAC Annual Reviews in Control, 2012. 36: p. 327-337.

18. ISO/IEC, Annex C: GERAM, in ISO/IS 15704:2000/Amd1:2005: Industrial automation systems - Requirements for enterprise-reference architectures and methodologies. 2005.

19. Rector, A., R. Qamar, and T. Marley, Binding Ontologies \& Coding systems to Electronic Health Records and Messages. Applied Ontology, 2009. 4(1).

20. Beale, T. and S. Heard, An Ontology-based Model of Clinical Information. S. Stud Health Technol Inform, 2007. 129 (Pt.1): p. 760-764.

21. Schulz, S., et al., SNOMED reaching its adolescence: Ontologists' and logicians' health check. Int J Med Inform, 2009. 78(1): p. 86-94.

22. Rosse, C. and J.V.L. Mejino, A reference ontology for biomedical informatics: the Foundational Model of Anatomy. J Biomed Inform., 2003. 36: p. 478-500.

23. Offodile, O.F. and L.L. Abdel-Malek, The virtual manufacturing paradigm: The impact of IT/IS outsourcing on manufacturing strategy. Int. J. Prod. Econ., 2002. 75(1-2): p. 147-159.

24. Mujber, T.S., T. Szecsi, and M.S.J. Hashmi, Virtual reality applications in manufacturing process simulation. J. Mater. Process. Technol., 2004. 155-156: p. 1834-1838.

25. De Vin, L.J., M. Holm, and A. Ng, The Information Fusion JDL-U model as a reference model for Virtual Manufacturing. Robot. Comput.-Integr. Manuf., 2010. 26(6): p. 629-638.

26. Goedkoop, M., et al., Introduction to LCA with SimaPro 7. 2008, Netherlands: Pré Consult.

27. Goedkoop, M., M. Oele, and S. Effting, SimaPro database manual methods library. 2004, Netherlands: Pré Consult.

28. Lanz, M. and R. Tuokko, Generic reference architecture for digital, virtual, and real representations of manufacturing systems, in Indo-US Workshop, Design-ing Sustainable Products, Services and Manufacturing systems. 2011: Indian Inst of Sci, Bangalore, India.

29. Ponce, P. and A. Molina, Fundamentos de LabVIEW. 2011: Alfaomega.

30. Ponce, P., et al., Intelligent Wheelchair and Virtual Training by LabVIEW. Lecture Notes in Computer Science, 2010. 6437: p. 422-435.

31. Ponce, P. and A. Molina, LabVIEW for Intelligent Control Research and Education, in Proceedings of the $4^{\text {th }}$ IEEE Int'l Conference on E-Learning in Industrial Electronics (ICELIE). 2010: Arizona, USA. p. 47-54.

32. Sarraipa, J., R. Jardim-Goncalves, and A. Steiger-Garcao, MENTOR: an enabler for interoperable intelligent systems. Int. J. of General Systems, 2010. 39(5): p. 557-573.

33. Jardim-Goncalves, R., et al., Knowledge Framework for Intelligent Manufacturing Systems. Journal of Intelligent Manufacturing, 2009. 22(5): p. 725-735.

34. Cretan, A., et al., NEGOSEIO: A Framework for Negotiations toward Sustainable Enterprise Interoperability. IFAC Ann. Rev. Ctrl., 2012. 36(2): p. 291-299.

35. Jardim-Goncalves, R., et al., Systematisation of Interoperability Body of Knowledge: the foundation for Enterprise Interoperability as a science. EIS, 2012. 6(3): p. 1-26.

36. Duan, L., et al., A negotiation framework for linked combinatorial optimization problems. Autonomous Agents and Multi-Agent Systems, 2012. 25(1): p. 158-182.

37. Grilo, A. and R. Jardim-Goncalves, Challenging electronic procurement in the AEC sector: A BIM-based integrated perspective. Automation in Construction, 2011. 20(2): p. 107-114.

38. Jardim-Goncalves, R., K. Popplewell, and A. Grilo, Sustainable interoperability: The future of Internet based industrial enterprises. Comp Ind, 2012. 63(8): p. 731-738. 